- The study looked at three different techniques to achieve a depth range of 0.4-0.6 mm consistently for veneer tooth reduction.

- The use of a small round bur to produce dimples as depth guides was found to be superior to two other tooth reduction techniques investigated; viz depth orientation grooves and free hand reduction.

- The results also showed inconsistent preparation depth even when depth gauging techniques, investigated in this study, were used. This was because either some of the depth guides themselves or the subsequent reduction of the tooth surface was inaccurate.

- Therefore, even when such depth gauging techniques are used, the clinician should employ great caution while finishing the tooth preparation. This is because, it is possible to over prepare while finishing the preparation even if the depth guides were accurate.

\title{
A study into the variations in the labial reduction of teeth prepared to receive porcelain veneers - a comparison of three clinical techniques
}

\author{
G. P. Cherukara, ${ }^{1}$ K. G. Seymour, ${ }^{2}$ D. Y. D. Samarawickrama ${ }^{3}$ and L. Zou ${ }^{4}$
}

\begin{abstract}
Objectives: Various techniques have been suggested to enable the operator to produce an even reduction of $0.5 \mathrm{~mm}$ of labial tooth enamel during preparation for a porcelain veneer. For example, in addition to the traditional free hand method, longitudinal or horizontal depth orientation grooves and the use of small round burs to produce dimples as depth guides have been suggested. However, there is no published data that compares how effective these techniques are at producing the 'ideal' veneer preparation. In this study three techniques were compared using the technique of co-ordinate metrology.
\end{abstract}

Method: A single operator using the above three techniques prepared 84 extracted teeth. Impressions of the prepared and unprepared teeth were scanned using a co-ordinate measuring machine (CMM).

Measurements of maximum labial reduction along the mid-labial plane were taken and analysed.

Results: The study showed that among the three techniques studied the use of small round burs (D001-012), when used side on at an angle of $45^{\circ}$ to the tooth surface to produce dimples as depth guides, resulted in the greatest frequency of tooth reductions closer to the 'ideal' depth chosen for this study, ie within the $0.4 \mathrm{~mm}-0.6 \mathrm{~mm}$ range.

Conclusion: The study concluded that even after using techniques designed to produce consistent preparations, a single operator still produced preparations with considerable variation from the ideal. The study showed that among the three techniques compared the use of small round burs, when used side on at an angle of $45^{\circ}$ to the tooth surface to produce dimples as depth guides, resulted in the greatest frequency of tooth reduction closer to the 'ideal' depth chosen for this study only, ie within the $0.4 \mathrm{~mm}-0.6 \mathrm{~mm}$ range. It is stressed that this range may not be the ideal in all clinical situations.

It is commonly accepted that, to satisfy the biologic needs of the periodontium, the technical needs of the ceramist and the aesthetic demands of the patient, some form of tooth preparation, confined within the enamel, is mandatory for veneering a tooth. ${ }^{1,2,3}$ Such a

${ }^{1 *} \mathrm{PhD}$ Student, ${ }^{2}$ Senior Lecturer, ${ }^{3}$ Senior Lecturer/Honorary Consultant, ${ }^{4}$ Dental Metrologist Department of adult oral health, Bart's and the London, Queen Mary's School of Medicine and Dentistry, London E1 2AD.

${ }^{*}$ Correspondence to: George Cherukara

Email:G.P.Cherukara@qmul.ac.uk

\section{Refereed paper}

Received 20.06.01; Accepted 14.11.01

๑ British Dental Journal 2002; 192: 401-404 preparation should also provide a veneer with good cervical and proximal adaptation. Mechanical reduction of enamel prior to acid etching has also been shown to increase the resin retention. ${ }^{4}$

Considering the thickness of enamel on the labial surface of anterior teeth and the minimum thickness of porcelain required for safe technical and clinical handling, a reduction of $0.5 \mathrm{~mm}$ of enamel is considered acceptable. However, it is the authors' considered opinion that such a decision to reduce the labial surface of the tooth by $0.5 \mathrm{~mm}$ should only be taken after careful individual patient and tooth assessment, thereby allowing for any pre-existing tooth surface loss. Several techniques have been advocated to help in an even reduction of enamel to $0.5 \mathrm{~mm}$ in addition to the traditional free hand method. These include the use of longitudinal ${ }^{5}$ or horizontal ${ }^{6}$ depth orientation grooves, small round burs to produce dimples as depth gauges, specially designed depth gauge burs ${ }^{7}$ (LVS-1 or LVS-2; Brasseler Laminate Veneer System Set 4151, Brasseler USA, Savannah, Ga.) and the use of plastic or putty indices. However, there is no published data that compares how effective these techniques are at producing the 'ideal' veneer preparation.

In spite of the advocation of the above techniques, many preparations fall outside the accepted guidelines. ${ }^{8}$ One of the reasons for this could be that clinicians do not make use of the various techniques suggested. ${ }^{8}$ However, we suspect that even if the suggested techniques and guidelines are followed, variations in tooth preparation result, as suggested by Nattress et al. ${ }^{9}$ Therefore, the aim of this study was to investigate the variations in reduction of teeth prepared by a single operator to receive porcelain veneers using three commonly used techniques:

- vertical depth orientation grooves

- free hand preparation

- dimples

It is also an attempt to address the issue of how best to achieve the desired depth of reduction of the tooth surface for a veneer restoration. A 3-dimensional co-ordinate measuring machine, with laser scanning probe, was used to provide data.

\section{MATERIALS AND METHODS}

Eighty-four extracted human upper central incisor teeth stored in formol saline were used in this study. Teeth were cleaned of extraneous deposits and divided into three groups of 28 teeth 
Figure 1 Labial impression of the tooth taken in an acrylic resin rectangular tray, with light-bodied polyvinyl silozane (blue coloured) impression material

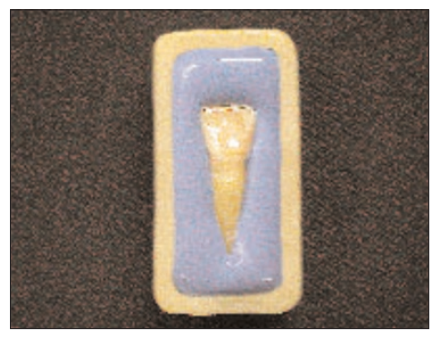

Figure 2 Pre- and postpreparation impressions ready to be scanned using CMM each. Impressions of the labial surfaces of the crowns and roots of these teeth were taken in rectangular acrylic resin boxes, measuring $35 \times 8 \times 5 \mathrm{~mm}$, using mid-blue, light-bodied polyvinyl siloxane impression material (Extrude, Kerr, Peterborough, UK), Figure 1. The teeth were mounted in dental plaster to provide better control during tooth preparation. A single operator (GPC) prepared teeth in each group, to receive porcelain veneers, using three different methods, namely: a) depth grooves, b) free hand and c) dimples.

a) In the first method, teeth were prepared by initially placing six vertical depth-orientation grooves on the labial surfaces, following the labial contour along the long axis of the tooth, with a straight fissure bur (R156-012). These depth grooves were eventually joined together with a chamfer finish bur (D198-022).

b) In the free hand technique, the labial surface was reduced using a chamfer finish bur (D198-022), arbitrarily to satisfy naked eye judgement of the preparation depth and quality.

c) With the third batch of teeth a small round bur (D001-012) was used, side on at an angle of $45^{\circ}$ to the labial surface of the tooth, to make dimples on that surface. The dimples were made on the teeth in even rows to fill the entire labial surface. Then a chamfer bur (D198-022) was used to prepare the surface, 'joining-up' the dimples.

Once the teeth were prepared, impressions of the prepared teeth were taken as earlier (Fig 2). These impressions, made before and after tooth preparation, were scanned uni-directionally by a co-ordinate measuring machine (Merlin 11, International Metrology Systems, Livingstone, UK) using a non-contact $830 \mathrm{~nm}$ wavelength laser triangulation probe (Renishaw 0P2, Renishaw, Gloucester, UK). The root surface area in the pre- and post-preparation impressions was used as the common area to superpose and plot the images constructed.
The maximum reduction along the mid-labial plane was then calculated and recorded using the pre-loaded software (Direct Computer Control Software Package, International Metrology Systems, UK; and Data Analysis Software Package ${ }^{10}$ ) by subtraction of these images. This group has used the co-ordinate measuring machine (CMM) previously, in studies relating to tooth preparation quality, with some success. ${ }^{11,12,13}$

\section{RESULTS}

Data collected from the scans, of the mid-labial region of the three groups of 'pre- and post-preparation' impressions, are given in Tables 1, 2 and 3. An example of a relevant profile is shown in Figure 3.

The greatest depth along the mid labial plane was identified and measured. Preparations that were greater than $0.4 \mathrm{~mm}$ and less than $0.6 \mathrm{~mm}$ were considered as 'ideal' for the purposes of this study. A simple comparison (Table 4) of the three groups of data revealed that teeth prepared with the free hand and depth orientation groove techniques resulted in greater frequency of deviation of tooth surface reduction from the 'ideal' preparation range of $0.4-0.6 \mathrm{~mm}$, as selected for this study. However, the use of dimples to prepare the labial surfaces resulted in greater frequency of preparations closer to this 'ideal'. Although the author was aware of the techniques used while measuring the depth, the metrologist who supervised was not aware of the technique or its significance. The decision regarding the deepest point of preparation was arrived on a consensual basis. Thus an attempt was made to avoid bias towards a technique at the measurement stage.

Analysis of variance (Table 5) also showed that there were statistically significant differences between the groups. However, the Bonferroni test (Table 6) revealed that this significant difference was only between group 1 and group 3 .

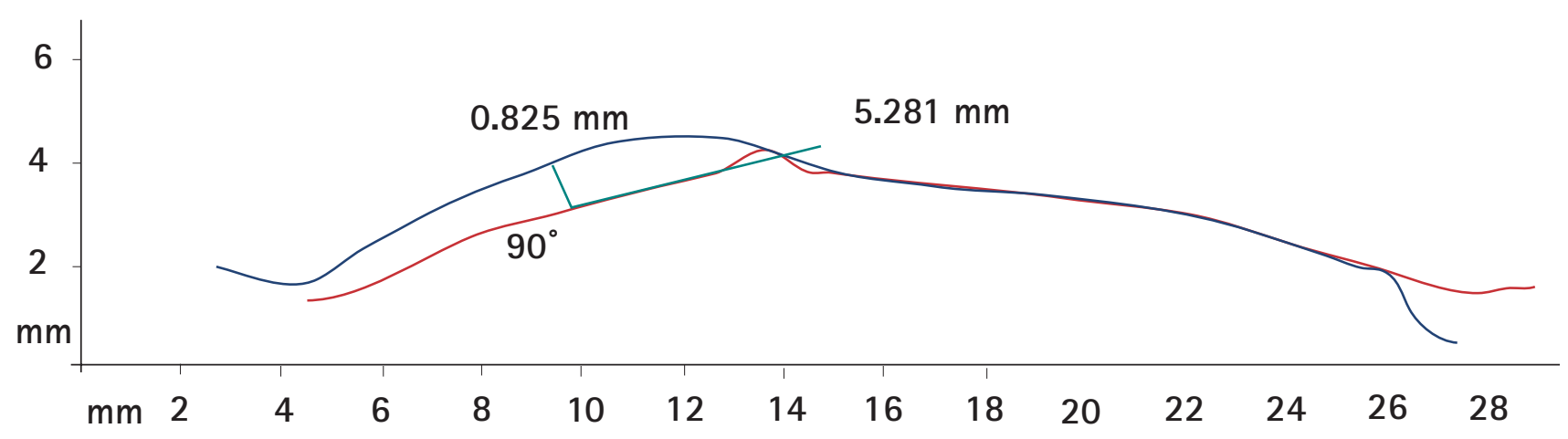




\begin{tabular}{ll}
$\begin{array}{l}\text { Table } 1 \text { Maximum mid-labial reduction of teeth } \\
\text { prepared with depth orientation grooves. }\end{array}$ \\
\hline \\
Tooth & Mid-labial reduction (mm) \\
\hline 1 & 0.707 \\
\hline 2 & 0.623 \\
\hline 3 & 0.447 \\
\hline 4 & 0.815 \\
\hline 5 & 0.787 \\
\hline 6 & 0.805 \\
\hline 7 & 0.439 \\
\hline 8 & 0.692 \\
\hline 9 & 0.825 \\
\hline 10 & 0.675 \\
\hline 11 & 0.771 \\
\hline 12 & 0.634 \\
\hline 13 & 0.842 \\
\hline 14 & 0.576 \\
\hline 15 & 0.535 \\
\hline 16 & 0.611 \\
\hline 17 & 0.651 \\
\hline 18 & 0.615 \\
\hline 19 & 0.735 \\
\hline 20 & 0.622 \\
\hline 21 & 0.794 \\
\hline 22 & 1.086 \\
\hline 23 & 0.659 \\
\hline 24 & 0.661 \\
\hline 25 & 0.492 \\
\hline 26 & 0.688 \\
\hline 27 & 0.839 \\
\hline 28 & 0.539 \\
\hline & \\
\hline Mean & 0.684 \\
\hline Randandard Deviation & 0.140 \\
\hline & 0.647 \\
\hline
\end{tabular}

Table 2 Maximum mid-labial reduction of teeth prepared with free hand

\begin{tabular}{|c|c|}
\hline Tooth & lid-labial reduction $(\mathrm{mm})$ \\
\hline 1 & 0.382 \\
\hline 2 & 0.488 \\
\hline 3 & 0.453 \\
\hline 4 & 0.805 \\
\hline 5 & 0.439 \\
\hline 6 & 0.692 \\
\hline 7 & 0.825 \\
\hline 8 & 0.675 \\
\hline 9 & 0.675 \\
\hline 10 & 0.675 \\
\hline 11 & 1.281 \\
\hline 12 & 0.633 \\
\hline 13 & 0.485 \\
\hline 14 & 0.542 \\
\hline 15 & 0.621 \\
\hline 16 & 0.619 \\
\hline 17 & 0.467 \\
\hline 18 & 0.769 \\
\hline 19 & 0.402 \\
\hline 20 & 0.792 \\
\hline 21 & 0.503 \\
\hline 22 & 0.667 \\
\hline 23 & 0.948 \\
\hline 24 & 0.508 \\
\hline 25 & 0.672 \\
\hline 26 & 0.918 \\
\hline 27 & 0.488 \\
\hline 28 & 0.615 \\
\hline Mean & 0.644 \\
\hline Standard Deviation & 0.196 \\
\hline Range & 0.899 \\
\hline Minimum & 0.382 \\
\hline Maximum & 1.281 \\
\hline
\end{tabular}

Table 3 Maximum mid-labial reduction of teeth prepared with dimples

\begin{tabular}{|c|c|}
\hline Tooth & id-labial reduction (mm) \\
\hline 1 & 0.638 \\
\hline 2 & 0.443 \\
\hline 3 & 0.375 \\
\hline 4 & 0.446 \\
\hline 5 & 0.568 \\
\hline 6 & 0.588 \\
\hline 7 & 0.723 \\
\hline 8 & 0.413 \\
\hline 9 & 0.483 \\
\hline 10 & 0.489 \\
\hline 11 & 0.590 \\
\hline 12 & 0.569 \\
\hline 13 & 0.581 \\
\hline 14 & 0.489 \\
\hline 15 & 0.460 \\
\hline 16 & 0.468 \\
\hline 17 & 0.692 \\
\hline 18 & 0.499 \\
\hline 19 & 0.522 \\
\hline 20 & 0.498 \\
\hline 21 & 0.599 \\
\hline 22 & 0.545 \\
\hline 23 & 0.482 \\
\hline 24 & 0.538 \\
\hline 25 & 0.600 \\
\hline 26 & 1.345 \\
\hline 27 & 0.423 \\
\hline 28 & 0.478 \\
\hline Mean & 0.555 \\
\hline Standard Deviation & 0.175 \\
\hline Range & 0.97 \\
\hline Minimum & 0.375 \\
\hline Maximum & 1.345 \\
\hline
\end{tabular}

\section{DISCUSSION}

Upper central incisors with no restorations on the labial aspect of the crown, were chosen for the study as they are the teeth most commonly restored with porcelain veneers, ${ }^{8,} 14$ and previous studies on tooth preparation for veneers have also used these teeth. ${ }^{8,}{ }^{9}$ These teeth also have a large, flatter labio-lingual width, as it was thought that the tooth preparation would be affected by teeth with smaller labio-lingual width or greater curvatures of the labial surface ie lower incisors, canines and premolars. The use of non-carious, unrestored teeth prevented the size and sites of disease or restorations from influencing the preparations carried out. Further, these teeth were set in an arch form of dental plaster, as suggested by the work of Seymour. ${ }^{12}$ In his study he concluded that having teeth set in an arch form gave the operators a closer representation of the spatial relationships present in the mouth. To reduce inter-operator variability, ${ }^{9}$ only a single operator performed the tooth preparations.

As reported in earlier studies ${ }^{11,12,13}$ the co-ordinate measuring machine provided data in a very graphic form, with the benefit of reproducibility. A reproducibility of $0.007 \mathrm{~mm}$ has been reported after repeated measurements of this type in previous studies. ${ }^{12}$

All previous studies using the $\mathrm{CMM}^{11,12,13}$ have held the impression material in $8 \times 8 \mathrm{~mm}$ brass square tubes. The use of rigid acrylic trays of larger dimensions in this study allowed for a larger region of the tooth to be scanned so that an adequate area was available to superpose the pre- and post-preparation images. An impression surface of mid-blue polyvinyl siloxane gave greater accuracy than other materials and colours while using the CMM. ${ }^{11,13}$

The CMM's analytical ${ }^{12}$ software allowed measurement of distance in $\mathrm{mm}$ to three decimal places $(1 \mu \mathrm{m})$. Hence data have been quoted at that level. The preloaded software (DCC, International Metrology Systems, UK, and Data Analysis Software Package) ${ }^{10}$ identified the mid-labial plane. Scanning of the impressions were done uni-directionally, as bi-directional scanning introduces distortion to the final image giving errors in measurement. ${ }^{12}$ Furthermore, the triangulation probe used in this study was of a noncontact type, so the technique was non-destructive. The pre- and post-preparation scans were superposed to analyse the preparation. Superpose is defined as 'to bring into the same position as to coincide' and implies a more mathematically precise overlaying of images than superimpose which is defined as 'to lay above or on top of'. ${ }^{15,16}$

In this study $0.4 \mathrm{~mm}-0.6 \mathrm{~mm}$ was chosen as the ideal preparation range to meet the practicality of the study. Therefore, preparations falling within this range were considered acceptable during data analysis. However, there is strong support in the literature for 


\begin{tabular}{|cc|}
\hline Table $\mathbf{4}$ Percentage of preparations within the $\mathbf{0 . 4} \mathbf{- 0 . 6} \mathbf{~ m m ~ r a n g e . ~}$ \\
\hline Technique of preparation & $\%$ \\
\hline Depth groove & 21.428 \\
\hline Free hand & 35.714 \\
\hline Dimple & 82.142 \\
\hline
\end{tabular}

\begin{tabular}{|c|c|c|c|c|c|}
\hline Source & D.F. & Sum of Squares & Mean Squares & FRatio & FProb. \\
\hline Between Groups & 2 & 0.2453 & 0.1226 & 4.1508 & 0.0192 \\
\hline Within Groups & 81 & 2.3934 & 0.0295 & & \\
\hline Total & 83 & 2.6387 & & & \\
\hline
\end{tabular}

\begin{tabular}{|c|c|c|c|c|}
\hline Mean & Group & Grp 3 & Grp 2 & Grp 1 \\
\hline 0.5551 & Grp 3 & & & \\
\hline 0.6442 & Grp 2 & & & \\
\hline 0.6845 & Grp 1 & * & & \\
\hline
\end{tabular}

$0.5 \mathrm{~mm}$ intra-enamel reduction clinically. This may be refuted by the finding of Ferrari et al, ${ }^{17}$ who reported that the mean thickness of enamel $2 \mathrm{~mm}$ incisal to the cemento-enamel junction of a central incisor was $0.4 \mathrm{~mm}$, hence a preparation $0.5 \mathrm{~mm}$ may not be confined entirely to the enamel. Such a finding should lead us to review the present guidelines for tooth preparation for porcelain veneers.

The results of the study showed that despite there being a single operator there was variability in consistency of preparation depth, even within the same group. Neither was consistent labial reduction achieved, despite the various methods used. Free hand tooth preparations showed the greatest variability. However, it was clear that the use of dimples as depth guides produced more preparations within the $0.4-0.6 \mathrm{~mm}$ range. Hence it appeared to be a more consistent technique. The reason for this consistency may lie in the fact that the shank of the small round bur provides a definite stop when used side on at $45^{\circ}$ to the tooth surface while placing the dimples on the tooth surface. Therefore, the placement of dimples improved the accuracy of the depth guides, which helps in the accurate reduction of the tooth surface. This limiting action of the shank of the bur was not noticed with the chamfer finish bur or the straight fissure bur. Hence the inaccuracy and variation, compounded by visual perception of the depth of preparation ${ }^{18}$ might have resulted. In addition, the consistency observed with the dimple method might have resulted from the fact that more of these dimples, were placed across the face of the labial surface of the tooth to fill the area. In the case of depth orientation grooves a maximum of only six groves were placed on the labial surface.

It was interesting to note that in each technique there was an out-lying measurement. It was not clear as to why this happened. It may be that whichever technique a clinician used, there is a possibility that things could go grossly wrong. Hence, one cannot blindly depend on any particular technique, rather give attention to detail at every step of tooth preparation. It is intriguing that the highest of these out-liers was in the dimple technique, which was otherwise the preferred technique of the three.

The difference identified between groups two and three in Table 4 was not found to be statistically significant at the 95\% confidence interval as demonstrated by the Bonferroni test, possibly because of a type 2 error. This outcome could be used to highlight the need for a judicious sample size selection rather than the selection of an arbitrary number. Similar studies have used a sample size of ten, often for convenience.

It was surprising that although various techniques have been advocated to aid tooth preparation, no attempts to compare them have been made in the literature at the time of this study. The consequences of inappropriate tooth reduction for veneers are:

1. Dentine exposure leading to dentine sensitivity and a susceptible bond to dentine.

2. An over-countered restoration leading to periodontal and aesthetic complications.

3. Technical difficulty in placing the margins of the veneer without adequate tooth preparation.

4. Absence of definite seating landmarks making proper seating of the veneer on the tooth surface for luting difficult.

\section{CONCLUSIONS}

It could be concluded from this study that, of the three techniques examined, the use of dimples as depth orientation resulted in achieving the theoretical ideal of $0.5 \mathrm{~mm}$ reduction of enamel, although a range of 0.4-0.6 mm was considered acceptable for this study, more often than the other two techniques tested. It can also be concluded that variation in tooth preparation results even when widely advocated guidelines and techniques are followed. The clinician should bear in mind this possibility and the consequences of this variability when preparing teeth to receive porcelain veneers.

Additionally, the fact that an enamel reduction of $0.5 \mathrm{~mm}$ may, in any case, expose dentine points us in the direction of a review of the present guidelines for tooth reduction for porcelain veneers.

1 Garber D A. Porcelain laminate veneers - to prepare or not to prepare? Compend Contin Educ Dent 1991: 12: 178-181.

2 Garber D A. Porcelain laminate veneers: ten years later. Part 1. Tooth preparation. J Esthet Dent 1993; 5: 56-62.

3 Garber D A, Goldstein R E, Feinman R A. Porcelain laminate veneers.; Quintessence Publishing Co. Chicago, 1988.

4 Jordan R E, Zuzuki M, Gwinnetta J, Hunter J. K. Restorations of fractured and hypoplastic incisors by the acid etch resin technique: A three year report. J Am Dent Assoc 1977: 95: 795-803.

$5 \quad$ Miller B J. Porcelain veneers. Dental Update 1987; 14: 381-390.

6 Weinberg LA. Tooth preparation for Porcelain veneers. New York State Dent J 1989. 55: 25-28.

7 Sheets G, Taniguchi. Advantages and limitations in the use of porcelain veneer restorations. J Prosthet Dent 1990; 64: 406-411.

8 Brunion PA, Rihmond S, Wilson N H F. Variation in the depth of preparation for porcelain laminate veneers. Euro J Prosthodont Rest Dent 1997; 5: 89-92.

9 Natttess B R, Youngson C C, Patterson C J W et al. An in vitro assessment of tooth preparation for porcelain veneer restorations. J Dent 1995; 23: 165-170.

10 Jovanovski V, Lynch E. Analysis of the morphology of oral structures from 3-D co-ordinate data. PhD thesis, University of London, 1998.

11 Seymour K, Samarawickrama D, Zou L, Lynch E. Shoulder dimensions and angles of bonded crown preparations. J Dent Res 1995; 74: 553, Abst 1219.

12 Seymour K G. Variations in the labial 'shoulder' geometry of teeth prepared to receive metal ceramic crowns. PhD thesis, University of London, 1998.

13 Lynch E, Zou L, Bedford J, Jovanovski V, Burke F. Measurement of lesions of primary root caries. J Dent Res 1993: 72: 742, Abst 447.

14 Pincus C R. Building mouth personality. J CalifState Dent Assoc 1938; 14: 125-129.

15 Jovanovski V, Zou L, Bedford J, Lynch E. Geometric superimposition of a sequence of digitised replicas. J Dent Res 1993; 72: 742, Abst 446.

16 Little W, Fowler H. W, Coulson J. The shorter oxford dictionary. 3rd ed. Oxford: Clarendon press, 1978.

17 Ferrari M, Patroni S, Balleri P. Measurement of enamel thickness in relation to reduction for etched laminate veneers. Int J Periodont Rest Dent 1992: 23: 407-413.

18 Dunne S M. The limitation of visual perception in restorative dentistry. Dental Update 1993; 20: 198-205. 